\title{
A Semigroup Proof for the Well-posedness of the Linearised Shallow Water Equations
}

\author{
Petra Csomós* and Johannes Winckler ${ }^{\dagger}$
}

March 6, 2017

\begin{abstract}
We present a derivation of the shallow water equations and their linearisation from basic physical principles, and show the well-posedness of the linearised problem. We work in a functional analytic framework and use the theory of operator semigroups.
\end{abstract}

\section{Introduction}

Efforts to describe the movement of gases and liquids through mathematical equations have a long history. The first significant contribution is due to Leonard Euler's work [4] in 1755, who presented an initial concept of classical hydromechanics with the so-called Euler equations being valid for incompressible and inviscid fluids. Between 1822 and 1845, Navier, SaintVenant, and Stokes added a viscosity term to Euler's model. In 2000, the Clay Mathematics Institute included the analysis of existence and regularity of solutions of the three-dimensional incompressible Navier-Stokes equations in the list of the seven Millennium problems. Presently, this problem is still open.

Shallow water equations represent a special case of Navier-Stokes equations for incompressible and inviscid fluids moving on the rotating planet as first proposed by Saint-Venant in 1871, see [9]. They played an important role in the first attempts to describe the atmosphere's largescale dynamics, see e.g. [2]. Numerical weather prediction models and ocean dynamical models, e.g. tsunami forecasting, are still based on them.

Instead of only referring to the shallow water equations as presented in the literature, we aim at giving an insight into their derivation. Next we linearise this equation and obtain the linearised shallow water equations, which is needed frequently when solving the problem numerically, see [1]. In order to show that the linearised version gives rise to a well-posed Cauchy problem, we use functional analysis and operator theory. In particular, we rewrite the equation as an abstract Cauchy problem on a Hilbert space and use the theory of strongly continuous operator

*Institute of Mathematics, Eötvös Loránd University, and MTA-ELTE Numerical Analysis and Large Networks Research Group, Pázmány Péter sétány 1/C, 1117 Budapest, Hungary, csomos@cs.elte.hu

${ }^{\dagger}$ Department of Mathematics, University of Tübingen, Auf der Morgenstelle 10, 72076 Tübingen, Germany, jowi@fa.uni-tuebingen.de 
semigroups (cf. [3]). An application of Stone's theorem yields that the corresponding operator generates a strongly continuous unitary group, hence, the problem is well-posed.

In Section 2 we introduce the shallow water equations and the linearised problem. Section 3 contains the proof of the well-posedness of the linearised problem and supplements Ushijima's result [6], [11] without viscosity terms but with a different boundary condition.

\section{Shallow water equations}

We first introduce shallow water equations as usually studied in the literature and deduce its linearisation. Our main reference is [8].

We consider the motion of a fluid over a bounded smooth domain $\Omega \subset \mathbb{R}^{2}$. The position of a fluid particle at time $t \geq 0$ is given by the point $(x, y, z) \in \Omega \times[0, H] \subset \mathbb{R}^{3}$ with maximal fluid height $H>0$, where direction $x$ shows to the East, direction $y$ to the North, and direction $z$ to the local zenith. The motion can be described by the velocities $u(t, x, y, z), v(t, x, y, z), w(t, x, y, z)$ in the directions $x, y, z$, respectively. The functions $u, v, w$ are continuously differentiable from $\mathbb{R}^{+} \times \Omega \times[0, H]$ to $\mathbb{R}$. We will also use the differentiable functions $p, \varrho: \mathbb{R}^{+} \times \Omega \times[0, H] \rightarrow \mathbb{R}$ representing the fluid's pressure and density, respectively. For notational simplicity we will drop the variables if it is clear on which variables these quantities depend. Our basic assumptions are the following.

Assumptions 2.1. (a) Shallow water assumption: The horizontal scale (e.g., diam $\Omega$ ) is much larger than the vertical height $H$ of the fluid. Physically this means that the horizontal velocities are much larger than the vertical one, that is, $u, v \gg w$.

(b) The domain $\Omega$ is on the surface of the planet at mid-latitudes, that is, $\varphi \approx 45^{\circ}$.

(c) The planet is rotating with an angular velocity $\omega$ (for Earth $\omega \approx 7.2921 \cdot 10^{-5} \frac{1}{s}$ ).

(d) The fluid is incompressible, which means that its density $\varrho$ is constant within a fluid parcel for all $t \geq 0$, that is, $\frac{\mathrm{d}}{\mathrm{d} t} \varrho=0$.

(e) The fluid is inviscid, that is, it is assumed to have no viscosity (the viscous forces are negligible compared to the inertial forces).

(f) The fluid is in a hydrostatic equilibrium, which means that the gravitational force is balanced by the vertical pressure gradient force.

(g) The atmospheric pressure is constant on the surface of the fluid.

(h) The Coriolis parameter $f=2 \omega \sin \varphi$ is constant (for Earth $f \approx 10^{-4} \frac{1}{s}$ ). This corresponds to the value at latitude $\varphi=45^{\circ}$. Thus, we approximate the rotation of the domain $\Omega$ as it were a plane touching the planet's surface at the line with latitude $\varphi=45^{\circ}$. This kind of approximation causes only a slight error in the $y$-direction.

We note that shallow waters are approximately in hydrostatic equilibrium, therefore, it is not a real constraint to suppose Assumption 2.1(f) together with Assumption 2.1(a). Assumptions 
2.1 enable us to construct a simple model describing the motion of the fluid in a lake, or even the large-scale dynamics of the atmosphere or the oceans on mid-latitudes.

We frequently use the following notions.

(i) The total mass of the fluid does not change in a closed system. This law can be expressed by the continuity equation

$$
\frac{\mathrm{d}}{\mathrm{d} t} \varrho+\varrho\left(\partial_{x} u+\partial_{y} v+\partial_{z} w\right)=0
$$

which can be reformulated, using Assumption 2.1(d), as

$$
\partial_{x} u+\partial_{y} v+\partial_{z} w=0 .
$$

(ii) Due to the flow of the medium, the position of a fluid particle depends on time. Therefore, the time derivative of any function $\Phi(t, x(t), y(t), z(t))$, depending on time and position, can be computed by using the chain rule:

$$
\frac{\mathrm{d}}{\mathrm{d} t} \Phi=\partial_{t} \Phi+\frac{\mathrm{d}}{\mathrm{d} t} x \cdot \partial_{x} \Phi+\frac{\mathrm{d}}{\mathrm{d} t} y \cdot \partial_{y} \Phi+\frac{\mathrm{d}}{\mathrm{d} t} z \cdot \partial_{z} \Phi=\partial_{t} \Phi+u \partial_{x} \Phi+v \partial_{y} \Phi+w \partial_{z} \Phi,
$$

which is called the material or convective derivative.

The shallow water equations can be derived from the equations of motion. Newton's second law states that the acceleration of a fluid particle is proportional to the net force $\vec{F}$ acting on the fluid particle, and the coefficient equals the mass of the fluid particle. By considering a fluid particle of unitary volume, Newton's second law implies that

$$
\frac{\mathrm{d}}{\mathrm{d} t} \vec{v}=\frac{1}{\varrho} \vec{F},
$$

where $\vec{v}=(u, v, w)^{\top}$ is the velocity vector and $\vec{F}$ is the sum of the following forces acting on the fluid particle of unitary volume.

(1) The sum $\vec{F}_{g}$ of the gravitational and the centrifugal forces is acting approximately downwards with an absolute value of $\varrho g$, where $g>0$ is the mean gravitational acceleration on the planet's surface (for Earth $g \approx 9.81 \frac{\mathrm{m}}{\mathrm{s}^{2}}$ ).

(2) The Coriolis force $\vec{F}_{C}$ acts due to the rotation of the planet, and can be computed as $\vec{F}_{C}=-2 \varrho(\vec{\omega} \times \vec{v})$, where $\vec{\omega}=(0, \omega \cos \varphi, \omega \sin \varphi)^{\top}$ is the angular velocity vector "felt" by the fluid particle on the surface of the planet. By Assumptions 2.1(a) and (f), the Coriolis force has the form $\vec{F}_{C}=\varrho(f v,-f u, 0)^{\top}$.

(3) The pressure gradient force $\vec{F}_{p}=-\left(\partial_{x} p, \partial_{y} p, \partial_{z} p\right)^{\top}$ constrains the fluid particle to move towards a position with lower pressure value $p(t, x, y, z)$.

By inserting the forces above into Newton's second law (2) and by taking into account Assumption 2.1(f), one obtains the following differential equations for $u, v, w$ :

$$
\left\{\begin{aligned}
\frac{\mathrm{d}}{\mathrm{d} t} u & =-\frac{1}{\varrho} \partial_{x} p+f v, \\
\frac{\mathrm{d}}{\mathrm{d} t} v & =-\frac{1}{\varrho} \partial_{y} p-f u, \\
\frac{\mathrm{d}}{\mathrm{d} t} w & =-\frac{1}{\varrho} \partial_{z} p-g=0 .
\end{aligned}\right.
$$


We are, however, interested in the height $h(t, x, y)$ of the fluid at each point $(x, y) \in \Omega$ and for all $t \geq 0$. The hydrostatic equilibrium, Assumption 2.1(f), implies that

$$
\begin{aligned}
\partial_{z} p & =-\varrho g \\
\int_{z}^{h(t, x, y)} \partial_{z} p \mathrm{~d} z^{\prime} & =-\int_{z}^{h(t, x, y)} \varrho g \mathrm{~d} z^{\prime} \\
p(t, x, y, z) & =p(t, x, y, h)+\varrho g(h(t, x, y)-z),
\end{aligned}
$$

where the surface pressure $p(t, x, y, h)$ is constant in all variables by Assumption 2.1(g). Then the pressure gradient is rewritten with $h$ as

$$
\partial_{x} p=\varrho g \partial_{x} h \quad \text { and } \quad \partial_{y} p=\varrho g \partial_{y} h .
$$

By substituting these formulae back into the first two equations of problem (3), one obtains the differential equations for $u$ and $v$ expressed in terms of the height $h$. In order to find the corresponding differential equation describing the time evolution of $h$ at each position, we need a physically meaningful connection between $h$ and the vertical velocity $w$.

Assumptions 2.2. We consider the following boundary conditions in the vertical direction $z$.

(a) At the bottom of the fluid we have $w(t, x, y, 0)=0$.

(b) At the surface of the fluid $w(t, x, y, h(t, x, y))=\frac{\mathrm{d}}{\mathrm{d} t} h(t, x, y)$ holds.

For the sake of simplicity we also suppose the following.

Assumption 2.3. Let the velocities $u$ and $v$ not depend on $z$, that is, be constant in the vertical direction.

We note that the assumption above could be avoided by taking the vertically averaged velocity field leading to the same system of equations. We further note that physical experiments suggest that taking an initial velocity field $(u, v)$ being constant in $z$ will remain so. Now, the boundary conditions $w(t, x, y, 0)=0$, the equality

$$
w(t, x, y, h)=\frac{\mathrm{d}}{\mathrm{d} t} h(t, x, y)=\partial_{t} h+u \partial_{x} h+v \partial_{y} h,
$$

and the continuity equation (1) imply that

$$
\begin{aligned}
\partial_{t} h & =w(t, x, y, h(t, x, y))-w(t, x, y, 0)-u \partial_{x} h-v \partial_{y} h \\
& =\int_{0}^{h(t, x, y)} \partial_{z} w(t, x, y, z) \mathrm{d} z-u \partial_{x} h-v \partial_{y} h \\
& =-\int_{0}^{h(t, x, y)}\left(\partial_{x} u+\partial_{y} v\right) \mathrm{d} z-u \partial_{x} h-v \partial_{y} h \\
& =-h\left(\partial_{x} u+\partial_{y} v\right)-u \partial_{x} h-v \partial_{y} h
\end{aligned}
$$

which is the differential equation for $h$. 
The shallow water equations then take the form

$$
\left\{\begin{array}{l}
\partial_{t} h=-u \partial_{x} h-v \partial_{y} h-h\left(\partial_{x} u+\partial_{y} v\right) \\
\partial_{t} u=-u \partial_{x} u-v \partial_{y} u-g \partial_{x} h+f v \\
\partial_{t} v=-u \partial_{x} v-v \partial_{y} v-g \partial_{y} h-f u
\end{array}\right.
$$

subject to certain initial and boundary conditions. System (4) is usually written in dimensionless form, whose derivation is presented in the Appendix:

$$
\left\{\begin{array}{l}
\partial_{t} h=-u \partial_{x} h-v \partial_{y} h-h\left(\partial_{x} u+\partial_{y} v\right), \\
\partial_{t} u=-u \partial_{x} u-v \partial_{y} u-c_{\mathrm{F}} \partial_{x} h+c_{\mathrm{R}} v \\
\partial_{t} v=-u \partial_{x} v-v \partial_{y} v-c_{\mathrm{F}} \partial_{y} h-c_{\mathrm{R}} u
\end{array}\right.
$$

with $c_{\mathrm{F}}=\frac{1}{\mathrm{Fr}^{2}}>0$ and $c_{\mathrm{R}}=\frac{1}{\mathrm{Ro}}>0$, where $\mathrm{Fr}>0$ and $\mathrm{Ro}>0$ denote the Froude and the Rossby numbers, respectively. The Froude number describes the ratio between the velocity of the zonal and the gravity waves, while the Rossby number characterises the ratio between the time scales of the Coriolis and the inertial forces. The typical value of the Rossby number for large-scale phenomena is 0.1 in the atmosphere and approximately 1 in the ocean. We note that for simplicity we use the same letters for the dimensionless new variables as before. The dimensionless formulation makes it possible to treat the system as being independent of the actual parameters, e.g., the planet itself, its gravitational acceleration, its angular velocity, and hence, the Coriolis parameter as well. Equations (5) describe many physical systems depending only on the constants $c_{\mathrm{F}}$ and $c_{\mathrm{R}}$.

System (5) can be written in a compact way as follows. Let the function $\mathcal{U}: \mathbb{R}^{+} \times \Omega \rightarrow \mathbb{R}^{3}$ be defined as

$$
\mathcal{U}(t, x, y)=\left(\begin{array}{l}
h(t, x, y) \\
u(t, x, y) \\
v(t, x, y)
\end{array}\right)
$$

Then the shallow water equations (5) read as

$$
\partial_{t} \mathcal{U}=\mathcal{B}(\mathcal{U}) \mathcal{U}
$$

with the operator matrix

$$
\mathcal{B}(\mathcal{U})=-\left(\begin{array}{ccc}
u \partial_{x}+v \partial_{y} & h \partial_{x} & h \partial_{y} \\
c_{\mathrm{F}} \partial_{x} & u \partial_{x}+v \partial_{y} & -c_{\mathrm{R}} \\
c_{\mathrm{F}} \partial_{y} & c_{\mathrm{R}} & u \partial_{x}+v \partial_{y}
\end{array}\right)
$$

for $\mathcal{U} \in\left(\mathrm{C}^{1}(\Omega)\right)^{3}$, where problem (6) is subject to the following initial and boundary conditions:

$$
\left\{\begin{array}{l}
\mathcal{U}(0, x, y)=\mathcal{U}_{0}(x, y), \quad(x, y) \in \Omega, \\
\left\langle\left(\begin{array}{l}
u \\
v
\end{array}\right), \mu\right\rangle_{\mathbb{R}^{2}}=0 \quad \text { on } \partial \Omega
\end{array}\right.
$$

where $\mathcal{U}_{0} \in(\mathrm{L}(\Omega))^{3}$ is a given function, $\mu \in \mathbb{R}^{2}$ denotes the normal vector of the boundary $\partial \Omega$ and $\langle\cdot, \cdot\rangle_{\mathbb{R}^{2}}$ denotes the inner product in $\mathbb{R}^{2}$. The boundary condition especially means that the velocity is parallel to the wall at each point on the boundary. 
The solution of nonlinear problems often requires the solution of their linearised version. Since nonlinear problems, like shallow water equation (5), are in general impossible to solve exactly, certain numerical methods are usually applied to obtain their approximative solution. Such numerical methods are, e.g., the exponential integrators based on the variation-of-constants formula and for which the (preferably) exact solution of the linearised problem is needed, see [5]. Therefore, we show next how to obtain the linearised problem.

The linearised shallow water equations originate from the linearisation of problem (6) around a basic state $\overline{\mathcal{U}}=(\bar{h}, \bar{u}, \bar{v})^{\top}$ with $\bar{u}, \bar{v} \in \mathbb{R}$ and $H_{1}<\bar{h}(x, y)<H$ for all $(x, y) \in \Omega$ and some $H_{1}>0$. Thus, one considers the solution $\mathcal{U}$ to the problem (6) as the sum of the basic state $\overline{\mathcal{U}}$ and some perturbation $\mathcal{U}_{*}=\left(h_{*}, u_{*}, v_{*}\right)^{\top}$ of it. We take the basic state as the equilibrium point of problem (6) (its existence will be shown later on), hence, we have

$$
\partial_{t} \overline{\mathcal{U}}=\mathcal{B}(\overline{\mathcal{U}}) \overline{\mathcal{U}}=0
$$

We note that the operator matrix $\mathcal{B}(\mathcal{U})$ can be split into the solution-independent part $\mathcal{L}$ and the solution-dependent part $\mathcal{N}(\mathcal{U})$. Then problem (6) reads as

$$
\partial_{t} \mathcal{U}=\mathcal{L} \mathcal{U}+\mathcal{N}(\mathcal{U}) \mathcal{U}
$$

with the operators

$$
\mathcal{L}=-\left(\begin{array}{ccc}
0 & 0 & 0 \\
c_{\mathrm{F}} \partial_{x} & 0 & -c_{\mathrm{R}} \\
c_{\mathrm{F}} \partial_{y} & c_{\mathrm{R}} & 0
\end{array}\right)
$$

and

$$
\mathcal{N}(\mathcal{U})=-\left(\begin{array}{ccc}
u \partial_{x}+v \partial_{y} & h \partial_{x} & h \partial_{y} \\
0 & u \partial_{x}+v \partial_{y} & 0 \\
0 & 0 & u \partial_{x}+v \partial_{y}
\end{array}\right)
$$

Inserting $\mathcal{U}=\overline{\mathcal{U}}+\mathcal{U}_{*}$ into the problem (9) leads to the terms

$$
\partial_{t}\left(\overline{\mathcal{U}}+\mathcal{U}_{*}\right)=\mathcal{L} \overline{\mathcal{U}}+\mathcal{L} \mathcal{U}_{*}+\mathcal{N}(\overline{\mathcal{U}}) \overline{\mathcal{U}}+\mathcal{N}(\overline{\mathcal{U}}) \mathcal{U}_{*}+N\left(\mathcal{U}_{*}\right) \overline{\mathcal{U}}+\mathcal{N}\left(\mathcal{U}_{*}\right) \mathcal{U}_{*}
$$

Relation (8) implies that

$$
\partial_{t} \overline{\mathcal{U}}=\mathcal{B}(\overline{\mathcal{U}}) \overline{\mathcal{U}}=\mathcal{L} \overline{\mathcal{U}}+\mathcal{N}(\overline{\mathcal{U}}) \overline{\mathcal{U}}
$$

therefore, problem (6) reduces to

$$
\partial_{t} \mathcal{U}_{*}=\mathcal{L} \mathcal{U}_{*}+\mathcal{N}(\overline{\mathcal{U}}) \mathcal{U}_{*}+\mathcal{N}\left(\mathcal{U}_{*}\right) \overline{\mathcal{U}}+\mathcal{N}\left(\mathcal{U}_{*}\right) \mathcal{U}_{*}
$$

Furthermore, we have

$$
\mathcal{N}\left(\mathcal{U}_{*}\right) \overline{\mathcal{U}}=-\left(\begin{array}{c}
u_{*} \partial_{x} \bar{h}+v_{*} \partial_{y} \bar{h}+h_{*} \partial_{x} \bar{u}+h_{*} \partial_{y} \bar{v} \\
u_{*} \partial_{x} \bar{u}+v_{*} \partial_{y} \bar{u} \\
u_{*} \partial_{x} \bar{v}+v_{*} \partial_{y} \bar{v}
\end{array}\right)=\mathcal{L}_{1} \mathcal{U}_{*}
$$


with

$$
\mathcal{L}_{1}=-\left(\begin{array}{ccc}
\partial_{x} \bar{u}+\partial_{y} \bar{v} & \partial_{x} \bar{h} & \partial_{y} \bar{h} \\
0 & \partial_{x} \bar{u} & \partial_{y} \bar{u} \\
0 & \partial_{x} \bar{v} & \partial_{y} \bar{v}
\end{array}\right) .
$$

By denoting $\mathcal{A}:=\mathcal{L}+\mathcal{N}(\overline{\mathcal{U}})+\mathcal{L}_{1}$, being independent of the solution, problem (10) has the form

$$
\partial_{t} \mathcal{U}_{*}=\mathcal{A} \mathcal{U}_{*}+\mathcal{N}\left(\mathcal{U}_{*}\right) \mathcal{U}_{*}
$$

subject to the initial and boundary conditions originating from that of problem (6):

$$
\left\{\begin{array}{l}
\mathcal{U}_{*}(0, x, y)=\mathcal{U}_{0}(x, y)-\overline{\mathcal{U}}, \quad(x, y) \in \Omega, \\
\left\langle\left(\begin{array}{l}
u_{*} \\
v_{*}
\end{array}\right), \mu\right\rangle_{\mathbb{R}^{2}}=0 \quad \text { on } \partial \Omega .
\end{array}\right.
$$

We note that the original boundary condition in (7) implies

$$
\left\langle\left(\begin{array}{c}
u_{*} \\
v_{*}
\end{array}\right), \mu\right\rangle_{\mathbb{R}^{2}}=-\left\langle\left(\begin{array}{l}
\bar{u} \\
\bar{v}
\end{array}\right), \mu\right\rangle_{\mathbb{R}^{2}} \text { on } \partial \Omega,
$$

however, for the semigroup proof we will need the orthogonality in (11). Furthermore, it leads to the orthogonality condition

$$
\left\langle\left(\frac{\bar{u}}{v}\right), \mu\right\rangle_{\mathbb{R}^{2}}=0 \text { on } \partial \Omega
$$

for the basic state, as well. The linear problem

$$
\partial_{t} \mathcal{U}_{\text {lin }}=\mathcal{A} \mathcal{U}_{\text {lin }}
$$

is called the system of linearised shallow water equations being subject to the initial and boundary conditions (11).

Our aim is to show that problem $(13),(11)$ has a unique solution only for an appropriate basic state, more precisely, only in the case when $\bar{u}, \bar{v}=0$ and $\bar{h} \in(0, H)$ is constant. At this point, however, we do not make any new assumptions on the basic state. Relation (8) yields then

$$
\begin{aligned}
& 0=\operatorname{div}\left(\bar{h}\left(\begin{array}{l}
\bar{u} \\
\bar{v}
\end{array}\right)\right), \\
& 0=c_{\mathrm{F}} \operatorname{grad} \bar{h}-c_{\mathrm{R}}\left(\begin{array}{c}
\bar{v} \\
-\bar{u}
\end{array}\right)+\left(\begin{array}{l}
\left\langle\left(\bar{u} \frac{\bar{u}}{\bar{v}}\right), \operatorname{grad} \bar{u}\right\rangle_{\mathbb{R}^{2}} \\
\left\langle\left(\begin{array}{l}
\bar{u} \\
\bar{v}
\end{array}\right), \operatorname{grad} \bar{v}\right\rangle_{\mathbb{R}^{2}}
\end{array}\right) .
\end{aligned}
$$

If $\bar{u}$ and $\bar{v}$ are constant, these formulae yield

$$
c_{\mathrm{F}} \operatorname{grad} \bar{h}=c_{\mathrm{R}}\left(\begin{array}{c}
\bar{v} \\
-\bar{u}
\end{array}\right)
$$

which describes the geostrophic balance, i.e., when the horizontal Coriolis force is balanced by the horizontal pressure gradient force. It fits well for the large-scale motions in the atmosphere and the oceans, and it fulfils problem (6). For $\bar{v}=0$, the basic state corresponds to the constant zonal west flow which dominates the weather at the mid-latitudes. The choice $\bar{u}=\bar{v}=0$ leads to $\bar{h} \in\left(H_{1}, H\right)$ constant meaning a basic state of rest. We will show that the problem (13) with initial and boundary conditions (11) is well-posed only in the last case. This corresponds to the physical meaning of the problem: It models the behaviour of a fluid over a bounded domain, hence, of a lake for instance. The choice of any basic state with $\bar{u} \neq 0$ or $\bar{v} \neq 0$ would describe a flow across the lakeside. 


\section{Well-posedness of the linear system}

This section is devoted to show how to rewrite problem (13) as an abstract Cauchy problem, and to prove that the operator appearing is the genarator of a strongly continuous semigroup. In that case the problem is well-posed. We will show that this applies only for the basic state of rest. In their works [6], [11], Kanamaya and Ushijima proved that the operator, containing viscous terms as well, is maximal dissipative on an appropriate Hilbert space, hence, it is a generator of a strongly continuous contraction semigroup. We will show the generator property by proving that the inviscid operator is skew-adjoint. Moreover, unlike the results above which take homogeneous Dirichlet boundary conditions for $u$ and $v$, we will take the boundary condition where the flow is parallel to the boundary on $\partial \Omega$ (especially, $u, v$ can vanish on $\partial \Omega$ ).

The operator $\mathcal{A}$ in problem (13) has the form

$$
\mathcal{A}=-\left(\begin{array}{ccc}
\bar{u} \partial_{x}+\bar{v} \partial_{y}+\partial_{x} \bar{u}+\partial_{y} \bar{v} & \bar{h} \partial_{x}+\partial_{x} \bar{h} & \bar{h} \partial_{y}+\partial_{y} \bar{h} \\
c_{\mathrm{F}} \partial_{x} & \bar{u} \partial_{x}+\bar{v} \partial_{y}+\partial_{x} \bar{u} & -c_{\mathrm{R}}+\partial_{y} \bar{u} \\
c_{\mathrm{F}} \partial_{y} & c_{\mathrm{R}}+\partial_{x} \bar{v} & \bar{u} \partial_{x}+\bar{v} \partial_{y}+\partial_{y} \bar{v}
\end{array}\right) .
$$

In order to show the well-posedness of system (13), we rewrite it into an abstract Cauchy problem

$$
\left\{\begin{aligned}
\frac{\mathrm{d}}{\mathrm{d} t} \boldsymbol{u}(t) & =A \boldsymbol{u}(t), \quad t>0 \\
\boldsymbol{u}(0) & =\boldsymbol{u}_{0}
\end{aligned}\right.
$$

on the Hilbert space $\left(\mathrm{L}^{2}(\Omega)\right)^{3}$, where the operator $A$ and its domain $D(A)$ are defined for all $\boldsymbol{u}=\left(u_{1}, u_{2}, u_{3}\right) \in D(A)$ as

$$
A \boldsymbol{u}=-\left(\begin{array}{c}
\bar{u} \partial_{x} u_{1}+\bar{v} \partial_{y} u_{1}+\bar{h}\left(\partial_{x} u_{2}+\partial_{y} u_{3}\right)+u_{1}\left(\partial_{x} \bar{u}+\partial_{y} \bar{v}\right)+u_{2} \partial_{x} \bar{h}+u_{3} \partial_{y} \bar{h} \\
c_{\mathrm{F}} \partial_{x} u_{1}+\bar{u} \partial_{x} u_{2}+\bar{v} \partial_{y} u_{2}-c_{\mathrm{R}} u_{3}+u_{2} \partial_{x} \bar{u}+u_{3} \partial_{y} \bar{u} \\
c_{\mathrm{F}} \partial_{y} u_{1}+c_{\mathrm{R}} u_{2}+\bar{u} \partial_{x} u_{3}+\bar{v} \partial_{y} u_{3}+u_{2} \partial_{x} \bar{v}+u_{3} \partial_{y} \bar{v}
\end{array}\right)
$$

with the domain

$$
D(A)=\left\{\boldsymbol{u}=\left(u_{1}, u_{2}, u_{3}\right) \in\left(\mathrm{L}^{2}(\Omega)\right)^{3}: u_{1} \in \mathrm{H}^{1}(\Omega), \operatorname{div}\left(\begin{array}{c}
u_{2} \\
u_{3}
\end{array}\right) \in \mathrm{L}^{2}(\Omega),\left\langle\left(\begin{array}{c}
u_{2} \\
u_{3}
\end{array}\right), \mu\right\rangle_{\mathbb{R}^{2}}=0\right\},
$$

where, as before, $\langle\cdot, \cdot\rangle_{\mathbb{R}^{2}}$ denotes the inner product in $\mathbb{R}^{2}$ and $\mu \in \mathbb{R}^{2}$ is the normal vector field of the boundary $\partial \Omega$.

Definition 3.1 ([3, Def. II.6.8]). On a Banach space $X$, the abstract Cauchy problem (16) associated to a closed operator $A: D(A) \subset X \rightarrow X$ is called well-posed if the following properties are satisfied.

(i) For every $\boldsymbol{u}_{0} \in D(A)$, there exists a unique solution $\boldsymbol{u}$ of problem (16).

(ii) The operator $A$ has dense domain $D(A)$.

(iii) For every sequence $\left(\left(\boldsymbol{u}_{0}\right)_{n}\right)_{n \in \mathbb{N}} \subset D(A)$ satisfying $\lim _{n \rightarrow \infty}\left(\boldsymbol{u}_{0}\right)_{n}=0$, one has $\lim _{n \rightarrow \infty} \boldsymbol{u}\left(t ;\left(\boldsymbol{u}_{0}\right)_{n}\right)=$ 0 uniformly for $t$ in compact intervals, where $\boldsymbol{u}\left(t ;\left(\boldsymbol{u}_{0}\right)_{n}\right)$ stands for the solution to problem (16) with the corresponding initial value $\left(\boldsymbol{u}_{0}\right)_{n}$. 
The next result shows that the well-posedness of a problem is strongly connected to the generator property of the operator.

Proposition 3.2 ([3, Cor. II.6.9]). For a closed operator $A: D(A) \subset X \rightarrow X$, the associated abstract Cauchy problem (16) is well-posed if and only if A generates a strongly continuous semigroup on $X$.

In order to prove the well-posedness of the linearised shallow water equations (6), we need a suitable Banach space $X$. For this purpose we introduce an inner product $\langle\cdot, \cdot\rangle_{X}$ in $\left(\mathrm{L}^{2}(\Omega)\right)^{3}$ as

$$
\langle\boldsymbol{u}, \boldsymbol{v}\rangle_{X}:=\left\langle\left(\begin{array}{l}
u_{1} \\
u_{2} \\
u_{3}
\end{array}\right),\left(\begin{array}{l}
v_{1} \\
v_{2} \\
v_{3}
\end{array}\right)\right\rangle_{X}:=\left\langle\left(\begin{array}{c}
c_{\mathrm{F}} u_{1} \\
\bar{h} u_{2} \\
\bar{h} u_{3}
\end{array}\right),\left(\begin{array}{c}
v_{1} \\
v_{2} \\
v_{3}
\end{array}\right)\right\rangle_{\left(\mathrm{L}^{2}(\Omega)\right)^{3}} .
$$

Since $0<H_{1}<\bar{h}(x, y)<H$ holds for all $(x, y) \in \Omega$ and $c_{\mathrm{F}}>0$ is constant, the inner product $\langle\cdot, \cdot\rangle_{X}$ is equivalent to $\langle\cdot, \cdot\rangle_{\left(\mathrm{L}^{2}(\Omega)\right)^{3}}$, hence, $X=\left(\left(\mathrm{L}^{2}(\Omega)\right)^{3},\langle\cdot, \cdot\rangle_{X}\right)$ is a Hilbert space. The generator property of $(A, D(A))$ follows now from the next two theorems.

Theorem 3.3 (Stone's Theorem, [10, Thm. A, Thm. B], both cited in [3, Thm. II.3.24]). Let the operator $A$ with domain $D(A)$ be a densely defined operator on the Hilbert space $X$. Then $A$ is the generator of a unitary group if and only if $A$ is skew-adjoint, that is, the identity $A^{*}=-A$ holds.

Our aim is to show that $D(A)$ is dense in $X$ and that the operator $A$ is skew-adjoint on the Hilbert space $X$. Then the theorem above implies that $A$ is the generator of a unitary group, hence, the linearised shallow water equations are well-posed. To show the properties above, we need the following result as well.

Theorem 3.4 (Thm. 6.2 in [7]). Let $n \in \mathbb{N}$, let $\Omega$ be a bounded Lipschitz domain in $\mathbb{R}^{n}$ and $\mathrm{H}_{0}^{1}(\Omega) \subset G \subset \mathrm{H}^{1}(\Omega)$. Let $\gamma$ denote the normal trace map. By setting the spaces

$$
\begin{aligned}
\mathrm{H}^{\operatorname{div}, n}(\Omega) & =\left\{r \in\left(\mathrm{L}^{2}(\Omega)\right)^{n}: \operatorname{div} r \in \mathrm{L}^{2}(\Omega)\right\}, \\
\mathrm{H}_{0}^{\operatorname{div}, n}(\Omega) & =\left\{r \in \mathrm{H}^{\operatorname{div}, n}(\Omega): \gamma r=0\right\} \\
D & =\left\{r \in \mathrm{H}^{\operatorname{div}, n}(\Omega):\langle\operatorname{div} r, q\rangle_{\mathrm{L}^{2}(\Omega)}+\langle r, \operatorname{grad} q\rangle_{\left(\mathrm{L}^{2}(\Omega)\right)^{n}}=0 \forall q \in G\right\},
\end{aligned}
$$

we obtain the following assertions.

(i) The set $D$ is a closed subspace of $\mathrm{H}^{\mathrm{div}, n}(\Omega)$ that contains $\mathrm{H}_{0}^{\mathrm{div}, n}(\Omega)$, i.e., $\mathrm{H}_{0}^{\mathrm{div}, n}(\Omega) \subset D \subset$ $\mathrm{H}^{\operatorname{div}, n}(\Omega)$.

(ii) When we identify $\mathrm{L}^{2}(\Omega)$ and $\left(\mathrm{L}^{2}(\Omega)\right)^{n}$ with their own duals, and we consider grad $\left.\right|_{G}$ as an unbounded operator mapping the dense subspace $G$ of $\mathrm{L}^{2}(\Omega)$ into $\left(\mathrm{L}^{2}(\Omega)\right)^{n}$, we have $\left.\operatorname{grad}\right|_{G} ^{*}=-\left.\operatorname{div}\right|_{D}$.

(iii) Let $G$ be closed in $\mathrm{H}^{1}(\Omega)$. Then $D=\mathrm{H}^{\mathrm{div}, n}(\Omega)$ if and only if $G=\mathrm{H}_{0}^{1}(\Omega)$, and $D=$ $\mathrm{H}_{0}^{\mathrm{div}, n}(\Omega)$ if and only if $G=\mathrm{H}^{1}(\Omega)$. 
Proposition 3.5. Suppose that $(\bar{h}, \bar{u}, \bar{v})$ satisfies (12) and (14),(15). Then the operator $A$ with domain $D(A)$ defined in (17) and (18), respectively, is the generator of a unitary group with respect to $\langle\cdot, \cdot\rangle_{X}$ if and only if $\bar{u}=\bar{v}=0$ and $\bar{h} \in\left(H_{1}, H\right)$ is constant.

Proof. We show that $(A, D(A))$ has dense domain in $X$ and is skew-adjoint on $X$. Then Theorem 3.3 implies the result.

In order to show the skew-adjointedness, we compute the following inner product for all $\boldsymbol{u}=$ $\left(u_{1}, u_{2}, u_{3}\right), \boldsymbol{v}=\left(v_{1}, v_{2}, v_{3}\right) \in D(A)$ (because of the real values inside, we do not mark the conjugation of the second component but the same calculations remain true for complex-valued function):

$$
\begin{aligned}
& \langle A \boldsymbol{u}, \boldsymbol{v}\rangle_{X}=\left\langle\left(\begin{array}{c}
c_{\mathrm{F}}(A \boldsymbol{u})_{1} \\
\bar{h}(A \boldsymbol{u})_{2} \\
\bar{h}(A \boldsymbol{u})_{3}
\end{array}\right),\left(\begin{array}{c}
v_{1} \\
v_{2} \\
v_{3}
\end{array}\right)\right\rangle_{\left(\mathrm{L}^{2}(\Omega)\right)^{3}} \\
= & -\int_{\Omega} v_{1}\left(c_{\mathrm{F}}\left(\bar{u} \partial_{x} u_{1}+\bar{v} \partial_{y} u_{1}\right)-c_{\mathrm{F}} \bar{h}\left(\partial_{x} u_{2}+\partial_{y} u_{3}\right)+c_{\mathrm{F}} u_{1}\left(\partial_{x} \bar{u}+\partial_{y} \bar{v}\right)+c_{\mathrm{F}} u_{2} \partial_{x} \bar{h}+c_{\mathrm{F}} u_{3} \partial_{y} \bar{h}\right) \\
& -\int_{\Omega} v_{2}\left(c_{\mathrm{F}} \bar{h} \partial_{x} u_{1}-\bar{h} \bar{u} \partial_{x} u_{2}-\bar{h} \bar{v} \partial_{y} u_{2}+c_{\mathrm{R}} \bar{h} u_{3}+\bar{h} u_{2} \partial_{x} \bar{u}+\bar{h} u_{3} \partial_{y} \bar{u}\right) \\
& -\int_{\Omega} v_{3}\left(c_{\mathrm{F}} \bar{h} \partial_{y} u_{1}-c_{\mathrm{R}} \bar{h} u_{2}-\bar{h} \bar{u} \partial_{x} u_{3}-\bar{h} \bar{v} \partial_{y} u_{3}+\bar{h} u_{2} \partial_{x} \bar{v}+\bar{h} u_{3} \partial_{y} \bar{v}\right) .
\end{aligned}
$$

For the sake of better understanding, we list the terms separately when applying Green's first identity. Due to (12) and $\boldsymbol{u}, \boldsymbol{v} \in D(A)$, we have

$$
\left\langle\left(\begin{array}{l}
u_{2} \\
u_{3}
\end{array}\right), \mu\right\rangle_{\mathbb{R}^{2}}=\left\langle\left(\begin{array}{c}
v_{2} \\
v_{3}
\end{array}\right), \mu\right\rangle_{\mathbb{R}^{2}}=\left\langle\left(\begin{array}{l}
\bar{u} \\
\bar{v}
\end{array}\right), \mu\right\rangle_{\mathbb{R}^{2}}=0 \text { on } \partial \Omega,
$$

therefore, the boundary integrals containing the terms above vanish, and the following terms remain:

$$
\begin{aligned}
& -\int_{\Omega}\left\langle c_{\mathrm{F}} v_{1}\left(\begin{array}{l}
\bar{u} \\
\bar{v}
\end{array}\right), \operatorname{grad} u_{1}\right\rangle_{\mathbb{R}^{2}}=\int_{\Omega} c_{\mathrm{F}} u_{1} v_{1} \operatorname{div}\left(\begin{array}{l}
\bar{u} \\
\bar{v}
\end{array}\right)+\int_{\Omega}\left\langle c_{\mathrm{F}} u_{1}\left(\begin{array}{l}
\bar{u} \\
\bar{v}
\end{array}\right), \operatorname{grad} v_{1}\right\rangle_{\mathbb{R}^{2}}, \\
& -\int_{\Omega} c_{\mathrm{F}} \bar{h} v_{1} \operatorname{div}\left(\begin{array}{l}
u_{2} \\
u_{3}
\end{array}\right)=\int_{\Omega}\left\langle c_{\mathrm{F}} \bar{h} \operatorname{div}\left(\begin{array}{l}
u_{2} \\
u_{3}
\end{array}\right), \operatorname{grad} v_{1}\right\rangle_{\mathbb{R}^{2}}+\int_{\Omega}\left\langle c_{\mathrm{F}} v_{1} \operatorname{div}\left(\begin{array}{l}
u_{2} \\
u_{3}
\end{array}\right), \operatorname{grad} \bar{h}\right\rangle_{\mathbb{R}^{2}}, \\
& -\int_{\Omega} c_{\mathrm{F}} u_{1} v_{1} \operatorname{div}\left(\begin{array}{l}
\bar{u} \\
\bar{v}
\end{array}\right), \\
& -\int_{\Omega}\left\langle c_{\mathrm{F}} v_{1}\left(\begin{array}{l}
u_{2} \\
u_{3}
\end{array}\right), \operatorname{grad} \bar{h}\right\rangle_{\mathbb{R}^{2}}, \\
& -\int_{\Omega}\left\langle c_{\mathrm{F}} \bar{h}\left(\begin{array}{l}
v_{2} \\
v_{3}
\end{array}\right), \operatorname{grad} u_{1}\right\rangle_{\mathbb{R}^{2}}=\int_{\Omega} c_{\mathrm{F}} \bar{h} u_{1} \operatorname{div}\left(\begin{array}{l}
v_{2} \\
v_{3}
\end{array}\right)+\int_{\Omega}\left\langle c_{\mathrm{F}} u_{1}\left(\begin{array}{l}
v_{2} \\
v_{3}
\end{array}\right), \operatorname{grad} \bar{h}\right\rangle_{\mathbb{R}^{2}}, \\
- & \int_{\Omega} c_{\mathrm{R}} \bar{h}\left(u_{2} v_{3}-v_{2} u_{3}\right), \\
- & \int_{\Omega}\left\langle\bar{h} v_{2}\left(\begin{array}{l}
\bar{u} \\
\bar{v}
\end{array}\right), \operatorname{grad} u_{2}\right\rangle_{\mathbb{R}^{2}}=\int_{\Omega} \bar{h} u_{2} v_{2} \operatorname{div}\left(\begin{array}{l}
\bar{u} \\
\bar{v}
\end{array}\right)+\int_{\Omega}\left\langle\bar{h} u_{2}\left(\begin{array}{l}
\bar{u} \\
\bar{v}
\end{array}\right), \operatorname{grad} v_{2}\right\rangle_{\mathbb{R}^{2}}+\int_{\Omega}\left\langle u_{2} v_{2}\left(\begin{array}{l}
\bar{u} \\
\bar{v}
\end{array}\right), \operatorname{grad} \bar{h}\right\rangle_{\mathbb{R}^{2}}, \\
- & \int_{\Omega}\left\langle\bar{h} v_{3}\left(\begin{array}{l}
\bar{u} \\
\bar{v}
\end{array}\right), \operatorname{grad} u_{3}\right\rangle=\int_{\Omega} \bar{h} u_{3} v_{3} \operatorname{div}\left(\begin{array}{l}
\bar{u} \\
\bar{v}
\end{array}\right)+\int_{\Omega}\left\langle\bar{h} u_{3}\left(\begin{array}{l}
\bar{u} \\
\bar{v}
\end{array}\right), \operatorname{grad} v_{3}\right\rangle_{\mathbb{R}^{2}}+\int_{\Omega}\left\langle u_{3} v_{3}\left(\frac{\bar{u}}{\bar{v}}\right), \operatorname{grad} \bar{h}\right\rangle_{\mathbb{R}^{2}},
\end{aligned}
$$




$$
-\int_{\Omega}\left\langle\bar{h} v_{2}\left(\begin{array}{l}
u_{2} \\
u_{3}
\end{array}\right), \operatorname{grad} \bar{u}\right\rangle_{\mathbb{R}^{2}}-\int_{\Omega}\left\langle\bar{h} v_{3}\left(\begin{array}{c}
u_{2} \\
u_{3}
\end{array}\right), \operatorname{grad} \bar{v}\right\rangle_{\mathbb{R}^{2}}
$$

By comparing these terms with the ones of $\langle\boldsymbol{u},-A \boldsymbol{v}\rangle_{X}$ (which are the same terms as above by changing the signs and the letters $u$ and $v$ ), one arrives at the identity

$$
\langle A \boldsymbol{u}, \boldsymbol{v}\rangle_{X}=\langle\boldsymbol{u},-A \boldsymbol{v}\rangle_{X}+(*),
$$

where $(*)$ denotes the sum of the following integrals:

$$
\begin{aligned}
& \int_{\Omega} \bar{h} u_{2} v_{2} \operatorname{div}\left(\frac{\bar{u}}{\bar{v}}\right)+\int_{\Omega}\left\langle u_{2} v_{2}\left(\begin{array}{l}
\bar{u} \\
\bar{v}
\end{array}\right), \operatorname{grad} \bar{h}\right\rangle_{\mathbb{R}^{2}}=\int_{\Omega} u_{2} v_{2} \operatorname{div}\left(\bar{h}\left(\frac{\bar{u}}{\bar{v}}\right)\right)=0 \text { due to }(14), \\
& \int_{\Omega} \bar{h} u_{3} v_{3} \operatorname{div}\left(\begin{array}{l}
\bar{u} \\
\bar{v}
\end{array}\right)+\int_{\Omega}\left\langle u_{3} v_{3}\left(\begin{array}{l}
\bar{u} \\
\bar{v}
\end{array}\right), \operatorname{grad} \bar{h}\right\rangle_{\mathbb{R}^{2}}=\int_{\Omega} u_{3} v_{3} \operatorname{div}\left(\bar{h}\left(\frac{\bar{u}}{\bar{v}}\right)\right)=0 \text { due to }(14), \\
& \int_{\Omega} c_{\mathrm{F}} u_{1} v_{1} \operatorname{div}\left(\begin{array}{l}
\bar{u} \\
\bar{v}
\end{array}\right), \\
& -\int_{\Omega}\left\langle\bar{h} v_{2}\left(\begin{array}{l}
u_{2} \\
u_{3}
\end{array}\right), \operatorname{grad} \bar{u}\right\rangle_{\mathbb{R}^{2}}-\int_{\Omega}\left\langle\bar{h} v_{3}\left(\begin{array}{c}
u_{2} \\
u_{3}
\end{array}\right), \operatorname{grad} \bar{v}\right\rangle_{\mathbb{R}^{2}}, \\
& -\int_{\Omega}\left\langle\bar{h} u_{2}\left(\begin{array}{c}
v_{2} \\
v_{3}
\end{array}\right), \operatorname{grad} \bar{u}\right\rangle_{\mathbb{R}^{2}}-\int_{\Omega}\left\langle\bar{h} u_{3}\left(\begin{array}{c}
v_{2} \\
v_{3}
\end{array}\right), \operatorname{grad} \bar{v}\right\rangle_{\mathbb{R}^{2}} .
\end{aligned}
$$

One can see that $(*)=0$ can only hold if $\bar{u}$ and $\bar{v}$ are constant. Since condition (12) means that the vector $(\bar{u}, \bar{v})$ should be parallel to the boundary at each point of $\partial \Omega$, this can only hold if $\bar{u}=\bar{v}=0$. Then (15) automatically implies that $\bar{h}$ is a constant value.

Hence, in the case of the basic state of rest, the identity $\langle A \boldsymbol{u}, \boldsymbol{v}\rangle_{X}=\langle\boldsymbol{u},-A \boldsymbol{v}\rangle_{X}$ holds for all $\boldsymbol{u}, \boldsymbol{v} \in D(A)$, which means that $-A$ is the adjoint of $A$ on $D(A)$. It remains to show that the maximal domain is already $D(A)$. We observe first that relation (19) and Theorem 3.4.(iii) implies that $D(A)=\mathrm{H}^{1}(\Omega) \times \mathrm{H}_{0}^{\text {div }, 2}(\Omega)$. Since $\mathrm{C}_{0}^{\infty}(\Omega)$ is dense in $\mathrm{H}^{1}(\Omega)$ with respect to the $\mathrm{L}^{2}(\Omega)$ norm, and $\left(\mathrm{C}_{0}^{\infty}(\Omega)\right)^{2}$ is dense in $\mathrm{H}_{0}^{\text {div,2}}(\Omega)$ with respect to the $\left(\mathrm{L}^{2}(\Omega)\right)^{2}$ norm, $D(A)$ is dense in $\left(\mathrm{L}^{2}(\Omega)\right)^{3}$. Moreover, for $n=2$ Theorem 3.4 implies that $\left.\operatorname{grad}\right|_{\mathrm{H}^{1}(\Omega)} ^{*}=-\left.\mathrm{div}\right|_{\mathrm{H}_{0}^{\mathrm{div}, 2}}$ from which it follows that $A^{*}=-A$. This completes the proof.

We remark that the standard linearisation procedure for quasilinear equations is to take the nonlinearity at a fixed value of the solution. If we take the basic state $\overline{\mathcal{U}}$ as the fixed value, we obtain the operator $\mathcal{A}_{1}:=\mathcal{L}+\mathcal{N}(\overline{\mathcal{U}})=\mathcal{A}-\mathcal{L}_{1}$ with which the equation does not necessary gives the linearisation about the basic state, therefore, we loose this kind of physical meaning of the solution (being the deviation from the basic state). We note that the computations for operator $\mathcal{A}_{1}$ give the same result as above.

\section{Appendix}

\section{Derivation of the dimensionless system (5)}

In order to rewrite system (4) into the dimensionless form (5), dimensionless new variables are to be defined. To this end we consider characteristic length $\mathbf{L}$, height $\mathbf{H}$, velocity $\mathbf{U}$, and 
time $\mathbf{T}=\mathbf{L} / \mathbf{U}$. Then all original variables $(t, x, y)$ and unknown functions $(h, u, v)$ can be transformed to their dimensionless counterparts $(\underline{t}, \underline{x}, \underline{y})$ and $(\underline{h}, \underline{u}, \underline{v})$ as

$$
\begin{aligned}
& t=\mathbf{T} \underline{t}=\frac{\mathbf{L}}{\mathbf{U}} \underline{t}, \quad x=\mathbf{L} \underline{x}, \quad y=\mathbf{L} \underline{y}, \\
& h=\mathbf{H} \underline{h}, \quad u=\mathbf{U} \underline{u}, \quad v=\mathbf{U} \underline{v} .
\end{aligned}
$$

The first equation in (4) is then rewritten by using these new variables as

$$
\begin{aligned}
\partial_{t} h & =-u \partial_{x} h-v \partial_{y} h-h\left(\partial_{x} u+\partial_{y}\right), \\
\frac{\mathbf{H U}}{\mathbf{L}} \partial_{\underline{t}} \underline{h} & =\frac{\mathbf{H U}}{\mathbf{L}}\left(-\underline{u} \partial_{\underline{x}} \underline{h}-\underline{v} \partial_{\underline{y}} \underline{h}-\underline{h}\left(\partial_{\underline{x}} \underline{u}+\partial_{\underline{y}} \underline{v}\right)\right), \\
\partial_{\underline{t}} \underline{h} & =-\underline{u} \partial_{\underline{x}} \underline{h}-\underline{v} \partial_{\underline{y}} \underline{h}-\underline{h}\left(\partial_{\underline{x}} \underline{u}+\partial_{\underline{y}} \underline{v}\right) .
\end{aligned}
$$

The second equation in (4) can be rewritten as

$$
\begin{aligned}
\partial_{t} u & =-u \partial_{x} u-v \partial_{y} u-g \partial_{x} h+f v, \\
\frac{\mathbf{U}^{2}}{\mathbf{L}} \partial_{\underline{t}} \underline{u} & =\frac{\mathbf{U}^{2}}{\mathbf{L}}\left(-\underline{u} \partial_{\underline{x}} \underline{u}-\underline{v} \partial_{\underline{y}} \underline{u}\right)-\frac{\mathbf{H}}{\mathbf{L}} g \partial_{\underline{x}} \underline{h}+f \mathbf{U} \underline{v}, \\
\partial_{\underline{t}} \underline{u} & =\left(-\underline{u} \partial_{\underline{x}} \underline{u}-\underline{v} \partial_{\underline{y}} \underline{u}\right)-\frac{g \mathbf{H}}{\mathbf{U}^{2}} \partial_{\underline{x}} \underline{h}+\frac{f \mathbf{L}}{\mathbf{U}} \underline{v} .
\end{aligned}
$$

The reformulation of the third equation is similar to that of the second, and one obtains

$$
\partial_{\underline{t}} \underline{v}=\left(-\underline{u} \partial_{\underline{x}} \underline{v}-\underline{v} \partial_{\underline{y}} \underline{v}\right)-\frac{g \mathbf{H}}{\mathbf{U}^{2}} \partial_{\underline{y}} \underline{h}-\frac{f \mathbf{L}}{\mathbf{U}} \underline{u} .
$$

By defining the Froude and the Rossby numbers, respectively, as

$$
\operatorname{Fr}:=\frac{\mathbf{U}}{\sqrt{g \mathbf{H}}}, \quad \text { Ro }:=\frac{\mathbf{U}}{f \mathbf{L}},
$$

and by dropping the notation for the dimensionless quantities, we obtain system (5).

\section{Acknowledgement}

The authors thank Klaus-Jochen Engel (L'Aquila) for his valuable suggestions during the work, and the anonymous referees for their comments which contributed to improving the quality of the manuscript. P.Cs. acknowledges the support of the National Research, Development, and Innovation Office (NKFIH) under the grant PD121117.

\section{References}

[1] Clancy, C. and Pudykiewicz, J. A., On the use of exponential time integration methods in atmospheric models. Tellus A 65, 20898 (2013), 1-16.

[2] Cullen, M. J. P., A Mathematical Theory of Large-Scale Atmosphere/ocean Flow. London: Imperial College Press 2006. 
[3] Engel, K.-J. and Nagel, R., One-Parameter Semigroups for Linear Evolution Equations. With contributions by S. Brendle, M. Campiti, T. Hahn, G. Metafune, G. Nickel, D. Pallara, C. Perazzoli, A. Rhandi, S. Romanelli and R. Schnaubelt. New York: SpringerVerlag 2000.

[4] Euler, L., Principes généraux du mouvement des fluides. Mémoires de l'académie des sciences de Berlin 11 (1757) 274-315.

[5] Hochbruck, M. and Ostermann, A., Exponential integrators. Acta Numerica 19 (2010) 209-286.

[6] Kanamaya, H., Ushijima, T., On the viscous shallow-water equations II - A linearized system. Bulletin of the University of Electro-Communications 1 (1988) 347-355.

[7] Kurula, M. and Zwart, H., The duality between the gradient and divergence operators on bounded Lipschitz domains. Department of Applied Mathematics, The University of Twente, Preprint 2012.

[8] Pedlosky, J., Geophysical Fluid Dynamics. New York and Berlin: Springer-Verlag 1982.

[9] de Saint-Venant, A. J. C. B, Théorie du mouvement non permanent des eaux, avec application aux crues des rivières et à l'introduction des marées dans leur lit. Comptes Rendus Acad. Sciences (1871) 147-154.

[10] Stone, M. H., On one-parameter unitary groups in Hilbert space. Ann. of Math. 33 (1932) 643-648.

[11] Ushijima, T., A semi-group theoretical analysis of a finite element method for a linearized viscous shallow-water system. Publ. Res. Inst. Math. Sci. 19 (1983) 1305-1328. 